\title{
VLBI detection of the HST-1 feature in the M 87 jet at $2 \mathrm{~cm}$
}

\author{
C. S. Chang ${ }^{1}$, E. Ros ${ }^{1,2}$, Y. Y. Kovalev ${ }^{3,1}$, and M. L. Lister ${ }^{4}$ \\ 1 Max-Planck-Institut für Radioastronomie, Auf dem Hügel 69, 53121 Bonn, Germany \\ e-mail: [cschang; ros] @mpifr.de \\ 2 Departament d'Astronomia i Astrofísica, Universitat de València, 46100 Burjassot, Spain \\ 3 Astro Space Centre of Lebedev Physical Institute, Profsoyuznaya 84/32, 117997 Moscow, Russia \\ e-mail: yyk@asc.rssi.ru \\ 4 Department of Physics, Purdue University, 525 Northwestern Avenue, West Lafayette, IN 47907, USA \\ e-mail: mlister@physics.purdue.edu
}

Received 18 December 2009 / Accepted 12 February 2010

\begin{abstract}
Context. A bright feature 80 pc away from the core in the powerful jet of M 87 shows highly unusual properties. Earlier radio, optical, and X-ray observations have shown that this feature, labeled HST-1, is superluminal, and is possibly connected with the TeV flare detected by HESS in 2005. It has been claimed that this feature might have a blazar nature because of these properties.

Aims. To examine whether HST-1 has a blazar-like nature, we analyzed $\lambda 2 \mathrm{~cm}$ VLBA archival data from dedicated full-track observations and the $2 \mathrm{~cm}$ survey/MOJAVE VLBI monitoring programs performed between 2000 and 2009.

Methods. We studied the subparsec scale structure of M 87 jet by using wide-field imaging techniques, after checking different weighting of the interferometric visibilities as a function of distance. The HST-1 region was imaged at milliarcsecond resolution.

Results. We present the first $2 \mathrm{~cm}$ VLBI detection of HST-1 in observations performed between early 2003 and early 2007, and analyze its evolution with time. Using the detections of HST-1, we find that the projected apparent speed is $0.61 \pm 0.31 c$. A comparison of the VLA and VLBA flux densities of this feature indicates that it is mostly resolved on milliarcsecond scales. This feature is optically thin $\left(\alpha \sim-0.8\right.$ for $\left.\mathrm{S} \propto \nu^{+\alpha}\right)$ between $\lambda 2 \mathrm{~cm}$ and $\lambda 20 \mathrm{~cm}$.

Conclusions. We do not find evidence that HST-1 has a blazar nature.
\end{abstract}

Key words. radio continuum: galaxies - techniques: high angular resolution - techniques: interferometric - galaxies: active galaxies: jets

\section{Introduction}

Active galactic nuclei (AGN) are among the most energetic phenomena in the Universe, which have been comprehensively studied since their initial discovery by Seyfert (1943). Although there is evidence that a supermassive black hole $(\mathrm{SMBH})$ is the engine launching the powerful jet (Urry \& Padovani 1995), the precise mechanism remains unknown.

One of the most widely studied AGN is M 87 (also known as Virgo A), a nearby elliptical galaxy located in the Virgo cluster. It hosts a very powerful one-sided jet emerging from the central region, and was the first extragalactic jet to be discovered (Curtis 1918). The synchrotron emission nature of the M 87 jet was first suggested by Baade (1956). Observations imply that M 87 contains $2.4 \times 10^{9} M_{\odot}$ within a $0.25^{\prime \prime}$ radius, which is indicative of a SMBH in its nucleus (Harms et al. 1994). Because of its proximity $\left(16.4 \mathrm{Mpc}, z=0.00436,1 \mathrm{mas}=0.08 \mathrm{pc}, 1 \mathrm{mas} \mathrm{yr}^{-1}=0.26 c\right.$, Jordán et al. 2005), M 87 is an ideal candidate for studying AGN phenomena, and has been monitored at many different wavelengths over several decades. In the observed one-sided jet of M 87 (Shklovsky 1964), superluminal motion was reported from Hubble Space Telescope (HST) observations within the innermost $6^{\prime \prime}$ of the jet with apparent speeds of $4 c$ to $6 c$ (Biretta et al. 1999). Discrepant speeds were reported from VLBA observations at $7 \mathrm{~mm}$ of values ranging between $0.25 c$ and $0.4 c$ (Ly et al. 2007), and a value of $2 c$ or even larger (Acciari et al. 2009). VLBA $\lambda 2 \mathrm{~cm}$ observations detected apparent speeds of $<0.05 \mathrm{c}$ from 1994 to 2007 (Kovalev et al. 2007; Lister et al. 2009b).
Therefore, the kinematical properties of the jet in M 87 remain an important topic of discussion.

In 1999, HST observations revealed a bright knot in the jet located $1^{\prime \prime}$ (projected distance of $0.08 \mathrm{kpc}$ ) away from the core. This feature, named HST-1, is active in the radio, optical, and X-ray regimes. VLBA $\lambda 20 \mathrm{~cm}$ observations showed that HST-1 has substructure and appears to contain superluminal components moving at speeds up to $4 c$ (Cheung et al. 2007). These observations suggest that HST-1 is a collimated shock in the AGN jet. Furthermore, multiwavelength observations were used to show that HST-1 could be related to the origin of the TeV emission observed in M 87 in 2005 by the HESS telescope (Aharonian et al. 2006). Comparing data taken in the near ultraviolet by Madrid (2009), soft X-rays (Chandra), and VLA $\lambda 2 \mathrm{~cm}$ observations (Cheung et al. 2007; Harris et al. 2006), the light curves of HST-1 reached a maximum in 2005, while the resolved core showed no correlation with the TeV flare. Therefore, the $\mathrm{TeV}$ emission from M 87 was proposed to originate in HST-1 (Harris et al. 2008). Based on those findings, Harris et al. (2008) suggest that HST-1 has a blazar nature. However, Acciari et al. (2009) reported rapid TeV flares from M 87 in February 2008, which probably originated in the core, instead of HST-1, which remained in a low state during the flares in 2008. The Fermi Large Area Telescope (LAT) team reported the detection of M 87 at gamma-ray energies (Abdo et al. 2009).

The AGN standard model considers the blazar behavior to originate in the vicinity of the SMBH. However, HST-1 is $80 \mathrm{pc}$ 
Table 1. Journal of VLBA $\lambda 2 \mathrm{~cm}$ observations of M $87^{a}$.

\begin{tabular}{|c|c|c|c|c|c|c|c|c|c|c|c|c|}
\hline \multirow[b]{2}{*}{ Epoch } & \multirow{2}{*}{$\begin{array}{l}\text { Exp. } \\
\text { Code }\end{array}$} & \multirow{2}{*}{$\begin{array}{c}t_{\text {int }} \\
\text { [min] }\end{array}$} & \multirow[t]{2}{*}{$N_{\mathrm{ant}}$} & \multicolumn{2}{|c|}{ Beam $^{c}$} & \multirow{2}{*}{$S_{\substack{\text { total } \\
\text { [Jy] }}}^{\mathrm{VLBA}, \mathrm{M} 87}$} & \multirow{2}{*}{$\begin{array}{c}S_{\text {peak,A }}^{\mathrm{M} 87} \\
{\left[\mathrm{Jy} \mathrm{beam}^{-1}\right]}\end{array}$} & \multirow{2}{*}{$\begin{array}{c}S_{\text {total }}^{\mathrm{HST}-1} \\
{[\mathrm{mJy}]}\end{array}$} & \multirow{2}{*}{$\begin{array}{c}S_{\text {peak,A }}^{\mathrm{HST}-1} \\
{\left[\mathrm{mJy} \mathrm{beam}^{-1}\right]}\end{array}$} & \multirow{2}{*}{$\begin{array}{c}S_{\text {peak,B }}^{\text {HST-1 }} \\
{\left[\mathrm{mJy} \mathrm{beam}^{-1}\right]}\end{array}$} & \multirow{2}{*}{$\begin{array}{c}\mathrm{rms}_{\mathrm{A}}{ }^{d} \\
{\left[\mu \mathrm{Jy}_{\text {beam }}{ }^{-1}\right]}\end{array}$} & \multirow{2}{*}{$\begin{array}{c}\mathrm{rms}_{\mathrm{B}}{ }^{d} \\
{\left[\mu \mathrm{Jy} \mathrm{beam}^{-1}\right]}\end{array}$} \\
\hline & & & & size[mas] & $\phi$ [degree] & & & & & & & \\
\hline 2000.06 & $\mathrm{BK} 073 \mathrm{~A}^{e, f}$ & 476 & 11 & $2.07 \times 1.35$ & -7 & 2.26 & 1.24 & $<1.36$ & $<0.26$ & $<0.23$ & 79 & 68 \\
\hline 2000.35 & $\mathrm{BK} 073 \mathrm{~B}^{e, f}$ & 476 & 11 & $1.88 \times 1.31$ & -13 & 2.33 & 1.29 & $<1.42$ & $<0.20$ & $<0.21$ & 70 & 71 \\
\hline 2000.99 & $\mathrm{BK} 073 \mathrm{C}^{e, f}$ & 476 & $10^{j}$ & $2.12 \times 1.52$ & -3 & 2.46 & 1.33 & $<1.84$ & $<0.25$ & $<0.33$ & 100 & 92 \\
\hline 2001.99 & $\mathrm{BR} Q 77 \mathrm{D}^{g, h}$ & 68 & 10 & $1.74 \times 1.09$ & -8 & 2.74 & 1.42 & $<5.24$ & $<0.66$ & $<0.70$ & 212 & 262 \\
\hline 2002.25 & $\mathrm{BR} 077 \mathrm{~J}^{g, h}$ & 57 & 10 & $1.77 \times 1.13$ & -6 & 2.54 & 1.40 & $<5.84$ & $<0.80$ & $<0.81$ & 300 & 292 \\
\hline 2003.09 & $\mathrm{BL} 111 \mathrm{E}^{g}$ & 54 & 10 & $1.85 \times 1.30$ & -8 & 2.85 & 1.57 & 3.98 & 1.01 & 2.13 & 218 & 206 \\
\hline 2004.61 & $\mathrm{BL}_{111 \mathrm{~N}^{g}}$ & 63 & 10 & $1.88 \times 1.16$ & -11 & 2.32 & 1.27 & 22.03 & 4.14 & 8.76 & 198 & 210 \\
\hline 2004.92 & $\mathrm{BL} 111 \mathrm{Q}^{g}$ & 64 & 10 & $1.95 \times 1.23$ & -14 & 2.41 & 1.39 & 23.59 & 2.70 & 9.97 & 223 & 295 \\
\hline 2005.30 & $\mathrm{BL} 123 \mathrm{E}^{g}$ & 63 & $9^{k}$ & $2.06 \times 1.37$ & -16 & 2.35 & 1.37 & 19.72 & 2.57 & 7.03 & 237 & 226 \\
\hline 2005.85 & $\mathrm{BL} 123 \mathrm{P}^{g}$ & 63 & 10 & $2.11 \times 1.25$ & -17 & 2.39 & 1.31 & 19.93 & 3.67 & 9.53 & 188 & 208 \\
\hline 2006.45 & $\mathrm{BL} 137 \mathrm{~F}^{g}$ & 22 & 10 & $1.85 \times 1.22$ & -10 & 2.51 & 1.51 & $<6.96$ & $<1.02$ & $<1.44$ & 295 & 348 \\
\hline 2007.10 & BL137N $\mathrm{N}^{g}$ & 42 & 10 & $1.95 \times 1.23$ & -13 & 2.67 & 1.53 & 10.49 & 1.14 & 2.94 & 201 & 255 \\
\hline 2007.42 & $\mathrm{BL}^{149 \mathrm{AA}^{g}}$ & 45 & 10 & $1.78 \times 1.25$ & -8 & 2.69 & 1.46 & $<7.70$ & $<0.81$ & $<1.13$ & 272 & 385 \\
\hline 2008.33 & BL149AO ${ }^{e}$ & 41 & $9^{j}$ & $1.77 \times 1.10$ & -7 & 3.24 & 1.79 & $<3.22$ & $<0.54$ & $<0.75$ & 193 & 161 \\
\hline 2009.10 & $\mathrm{BL}^{149 B \mathrm{BG}^{i}}$ & 50 & $9^{l}$ & $2.25 \times 1.52$ & -12 & 1.99 & 1.13 & $<5.26$ & $<0.36$ & $<0.49$ & 223 & 263 \\
\hline
\end{tabular}

Notes. ${ }^{(a)}$ Observations are in dual-polarization mode, except as indicated. ${ }^{(b)}$ Total scheduled VLBI on-source time. ${ }^{(c)}$ Beam A: natural weighting and tapering of Gaussian full-width half maximum 0.3 at $200 \mathrm{M} \lambda .{ }^{(d)}$ Root-mean-square image noise using natural weighting and tapering with a Gaussian factor of 0.3 at a radius of $200 \mathrm{M} \lambda .{ }^{(e)}$ Recording rate: $256 \mathrm{Mbit} \mathrm{s}^{-1}, 2$ bits per sample. ${ }^{(f)}$ Dedicated full-track experiment on M 87 (see Kovalev et al. 2007); the array included a single VLA antenna (Y1). ${ }^{(g)}$ Recording rate: 128 Mbit s$^{-1}, 1$ bit per sample. ${ }^{(h)}$ Single polarization hand (LL) observations only. ${ }^{(i)}$ Recording rate: $512 \mathrm{Mbit} \mathrm{s}^{-1}, 2$ bits per sample. ${ }^{(j)}$ Antenna NL missing. ${ }^{(k)}$ Antenna SC missing. ${ }^{(l)}$ Antenna HN missing.

away from the core. If the HST-1 blazar hypothesis were true, this would pose a challenge to current AGN models. In this paper, we examine this hypothesis using $\lambda 2 \mathrm{~cm}$ VLBI wide-field imaging of the HST-1 feature. We describe our VLBI observations and the corresponding data reduction in Sect. 2, the results are presented in Sect. 3, and the discussion is presented in Sect. 4. Finally, a short summary is presented in Sect. 5. Throughout this paper, we use the term "core" as the apparent origin of AGN jets that commonly appear as the brightest features in VLBI radio images of blazars (Lobanov 1998; Marscher 2008). We adopt a cosmology with $\Omega_{\mathrm{m}}=0.27, \Omega_{\Lambda}=0.73$, and $H_{0}=71 \mathrm{~km} \mathrm{~s}^{-1} \mathrm{Mpc}^{-1}$ (Komatsu et al. 2009).

\section{VLBI observations and data analysis}

M 87 has been monitored at $\lambda 2 \mathrm{~cm}$ with the VLBA since 1994 by the $2 \mathrm{~cm}$ Survey/MOJAVE programs ${ }^{1}$ (Kellermann et al. 2004; Lister et al. 2009a). We reanalyzed 12 epochs of these monitoring program data obtained after late 2001, and three observing sets of targeted observations on M 87 in 2000 (see Table 1). The $2 \mathrm{~cm}$ Survey/MOJAVE epochs from 2001 to 2009 were snapshot observations with a total integration time from 20 minutes to one hour, and were broken up into about six-minute-long scans to ensure wider $(u, v)$ coverage; the three epochs in 2000 were full-track observations with eight-hour integration times and included a single VLA antenna (Y1).

The VLBA data were processed by following the standard procedures in the AIPS cookbook, as described in detail by Kovalev et al. (2007) and Lister et al. (2009a). The data were fringe-fitted before the imaging process. The data from MOJAVE project epochs after 2007.61 were processed using the pulse calibration signals to align the phases instead of fringe fitting, because the positions of the sources and the VLBA antennas are well determined (Petrov et al. 2008, 2009), and the station clocks are known to be stable. However, for our wide-field imaging purposes, fringe fitting improved the accuracy of the

1 http://www.physics.purdue. edu/MOJAVE/ measured phase and rate across the observed band, and therefore the quality of the imaging of the extended jet of M 87. It also ensures the homogeneity of all the datasets.

To accurately image HST-1 with VLBI, we need to consider two issues. First, HST-1 lies $\sim 800 \lambda 2 \mathrm{~cm}$ beamwidth away from the brightest feature (the VLBA core). For this reason, time or frequency data averaging would cause time and bandwidth smearing in the HST-1 region. Second, based on earlier VLBA $\lambda 20 \mathrm{~cm}$ and VLA $\lambda 2 \mathrm{~cm}$ observations of HST- 1 (Cheung et al. 2007), we expect the total $2 \mathrm{~cm}$ flux density to be at the milliJansky level, which is much weaker than the total flux density of the inner jet $(\sim 2.5 \mathrm{Jy})$. To detect HST-1, we need to image the inner jet region and its extended structure (over tens of milliarcseconds), otherwise, the sidelobes from the core will cover the HST-1 emission. To reach this goal, we applied natural weighting and $(u, v)$-tapering to the whole dataset.

First, we averaged the data 16 -sec in time and averaged all channels in each IF in frequency, and obtained the inner jet initial model by applying phase and amplitude self-calibration using DIFMAP. The time-smearing effect of 16 seconds becomes significant at a distance of 200 mas from the field center, which is beyond the inner jet scale, and does not affect our data analysis at this stage. When the inner jet model was reasonably accurate, we applied the obtained CLEAN-components to the unaveraged data for the first amplitude-and-phase self-calibration using AIPS. Natural weighting and tapering with a Gaussian factor of 0.3 at a radius of $200 \mathrm{M} \lambda$ in the $(u, v)$-plane was applied to the whole dataset, and the resultant beam sizes are shown in Table 1. If we remove tapering and apply uniform weighting, the inner jet is more clearly resolved but a lower flux density is recovered. Therefore, the image noise level would be too high to detect the HST-1 feature.

To identify the extended component more clearly, and since full-resolution, uniform-weighting imaging did not improve the detection of this feature, we chose the approach of tapering and natural weighting. Furthermore, we downgraded the resolution to a larger beam size of $8 \times 3.4$ mas in PA $0^{\circ}$. This beam size 
was chosen specifically to allow comparison with the VLBA $\lambda 20 \mathrm{~cm}$ observations of Cheung et al. (2007). In the following sections, we label the smaller beams beam A, noting that the beam sizes differ slightly between epochs. These are listed in Table 1 . Beam $B$ refers to the beam size of $8 \times 3.4$ mas.

\section{Results}

We completed the wide-field imaging of 15 epochs of M 87 VLBA $\lambda 2 \mathrm{~cm}$ obtained between 2000 and 2009. Each epoch was imaged using beam A and beam B (see Sect. 2), and for each beam size, we applied two image cleaning fields, one for the M 87 inner jet region, and another for the HST-1 region, which was phase-shifted by -788.5 mas in right ascension and 348.9 mas in declination. We performed deep cleaning iterations until the image rms reached the expected thermal noise value. The rms values of the final images are listed in Table 1 . Three epochs in the year 2000 are full-track observations with an onsource time of 476 minutes, and have the lowest rms.

\subsection{Imaging of the inner jet of M87}

With tapering and natural weighting of the data, the extended inner jet was imaged over a region 100-200 mas in size, which varied according to the different sensitivity of each epoch. The inner jet images are shown in Fig. 1 (beam A) and Fig. 2 (beam B). The inner jet structure has an average total flux density of $\sim 2.5 \mathrm{Jy}$, exhibiting overall flux density changes up to 1 Jy during 2000 to 2009, as shown in Fig. 3. We estimated the error in flux densities to be $5 \%$, based on the typical amplitude calibration accuracy of the VLBA (Kovalev et al. 2005; Ulvestad \& Wrobel 2009). In February 2008, very-high-energy multiple flares were detected by the multiwavelength campaign of HESS, MAGIC, VERITAS, while VLBA $\lambda 7 \mathrm{~mm}$ observations detected a flare at the VLBA core simultaneously (Acciari et al. 2009). We also detected the 2008 flare from the core in our VLBA $\lambda 2 \mathrm{~cm}$ data, and therefore confirmed this result (see Fig. 3).

The structure of the inner jet changed during the period of our observations. However, the study of the inner jet is beyond the scope of this paper (see e.g., Kovalev et al. 2007; Walker et al. 2008; Lister et al. 2009b, for a detailed discussion of the structure and kinematics of the inner jet).

\subsection{Imaging of the HST-1 region}

We detected HST-1 in six of the 15 epochs that we analyzed from 2003 to early 2007 (detection limit: $5 \sigma$ ). As discussed in Sect. 4, this feature was too faint in most epochs, and the image noise was too high for its detection. Figure 4 shows hybrid maps of this region. The HST-1 images produced using beam A show that the brightest component has a size of $\sim 10$ mas down to the lowest contours, while the images produced using beam B reveal an extended structure $\sim 50$ mas in size, which is comparable to its size in VLBA $\lambda 20 \mathrm{~cm}$ observations (Cheung et al. 2007). The feature has a total flux density that varies between 4 and $24 \mathrm{mJy}$ (Fig. 5). The peak surface brightness varies from 1 to $4 \mathrm{mJy}^{-1}$ beam ${ }^{-1}$ (beam A) and 2 to $10 \mathrm{mJy} \mathrm{beam}^{-1}$ (beam B), as shown in Fig. 6. Epochs in which no detection is achieved are marked as upper limits (inverted triangles) in Figs. 5 and 6.

In 2003.09, HST-1 is marginally detected with both beam sizes; the brightness peak is $\sim 1 \mathrm{mJy}$ beam $^{-1}$, and the total flux density is $3.7 \mathrm{mJy}$. During 2004.61-2005.85, significant detections of HST-1 are achieved by beam A and beam B for 4 epochs,

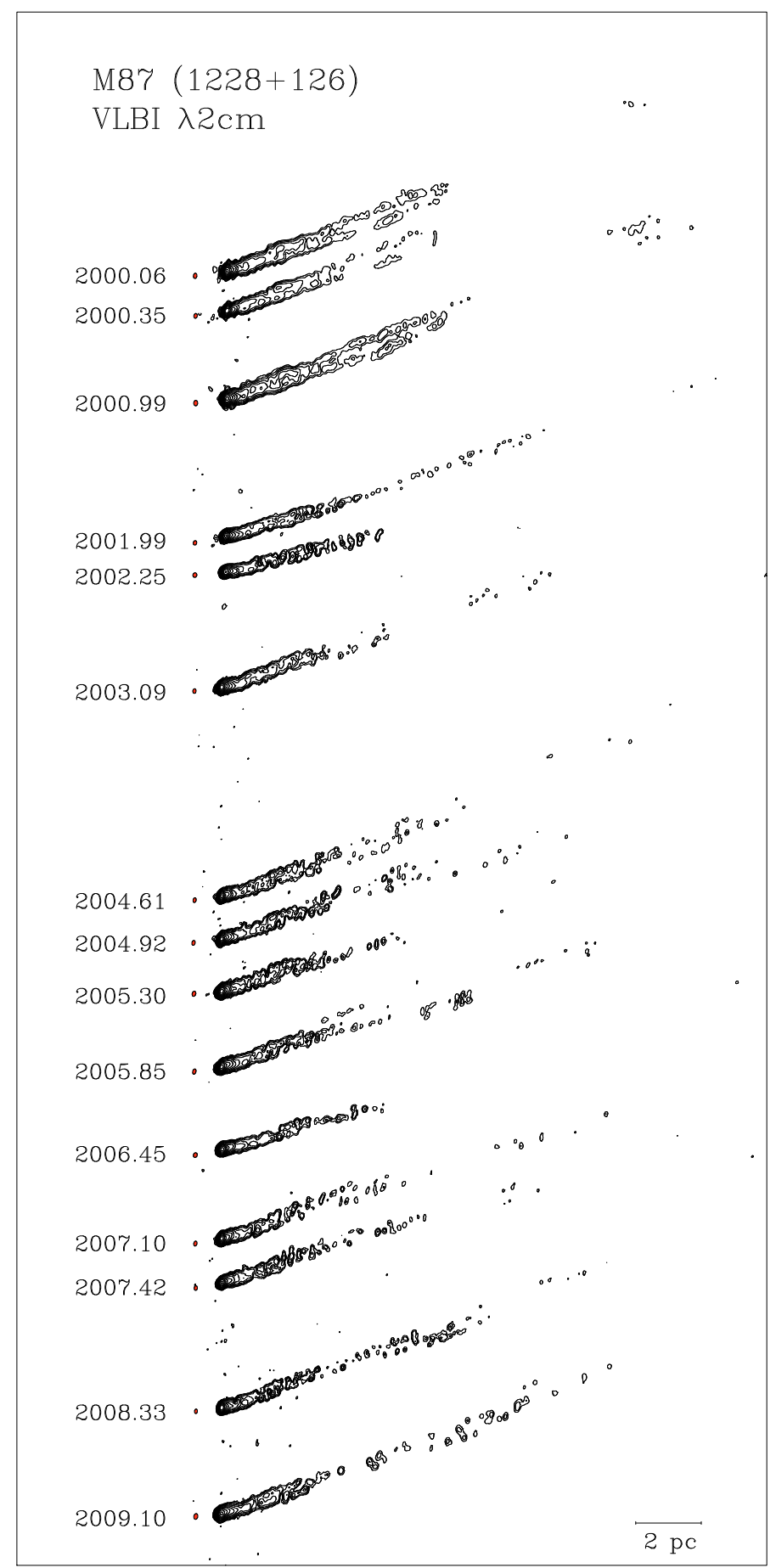

Fig. 1. Images of the central 220 mas of the M 87 jet (beam A, resulting from a $(u, v)$-taper of Gaussian 0.3 at $200 \mathrm{M} \lambda$ and natural weighting), spaced by their relative time intervals. The images are plotted with the same size scale. The contour levels all increase by factors of $\sqrt{2}$, and the lowest contours are (from 2000.06 to 2008.33 ) 0.5, 0.7, 0.5, 1.1, 1.3, $0.9,0.9,0.9,0.9,0.9,1.1,1.0,1.2,0.9,0.9 \mathrm{mJy}_{\text {beam }}^{-1}$, respectively. The restoring beams and brightness peak values are given in Table 1.

where the peak component remains dominant, and the total flux density fluctuation is $\sim 30 \%$. In 2007, the structure of HST-1 becomes more extended and complex, reaching a total flux density of $10 \mathrm{mJy}$ and developing two distinct peaks (peak a and peak b in Fig. 4).

For the epochs in which there were no detections, we derived upper limits to the total VLBI flux density of HST-1, based on 


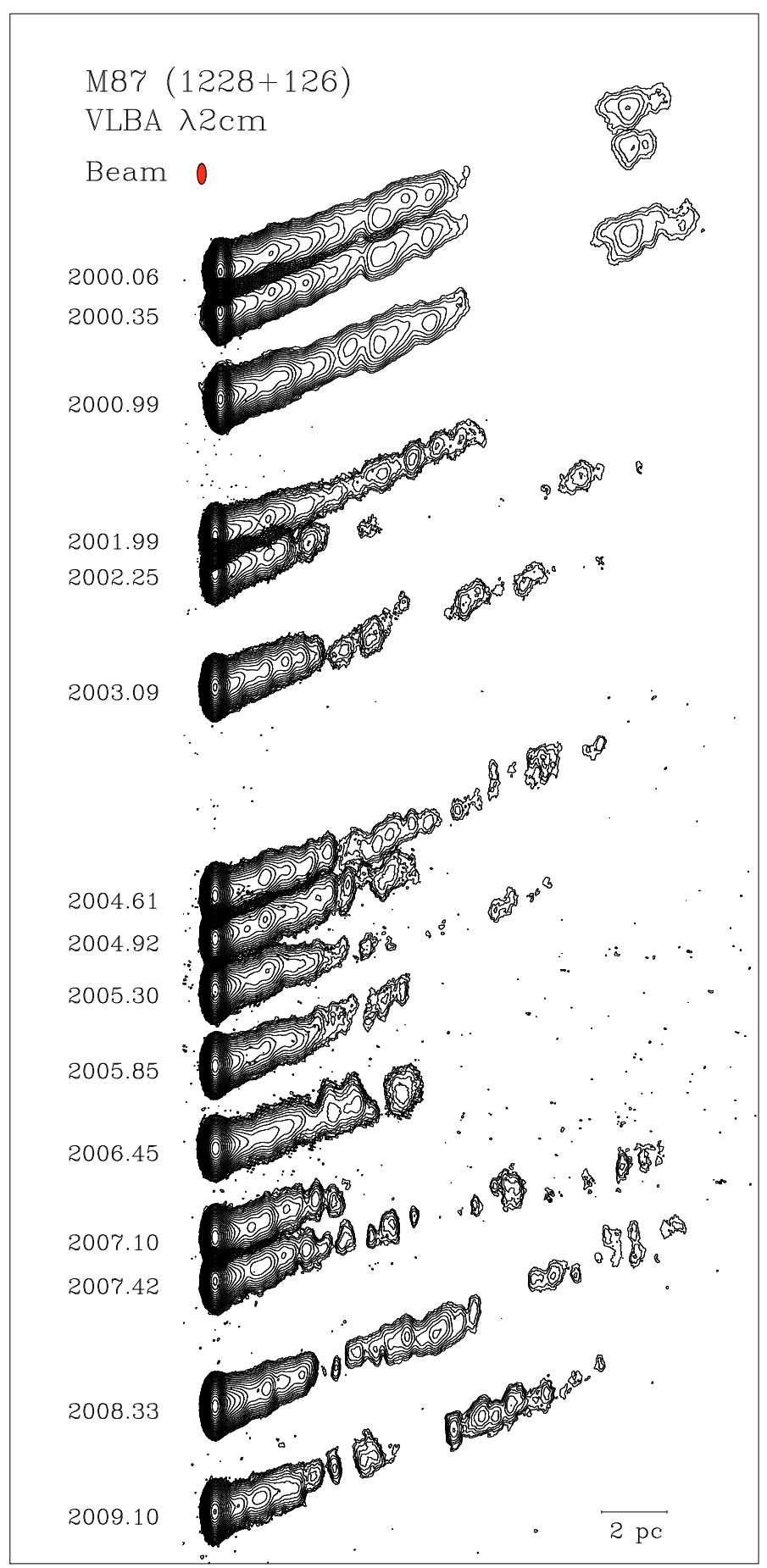

Fig. 2. Downgraded resolution images of the central 220 mas of the M 87 jet with beam B $(8 \times 3.4$ mas $)$, spaced by their relative time intervals. The contour levels all increase by factors of $\sqrt{2}$, and the lowest contours are (from 2000.06 to 2008.33) 0.5, 0.7, 0.5, 1.1, 1.3, 0.9, 0.9, $0.9,0.9,0.9,1.1,1.0,1.2,0.9,0.9 \mathrm{mJy} \mathrm{beam}^{-1}$, respectively.

the rms noise values of each epoch. The maximum amount of flux density hidden beneath the noise would be the rms value times the size of the HST-1 emission region (in units of beam size). Based on our HST-1 detection, we assume that the size of the HST-1 emission region is about 20 times the beam size, and we derived accordingly the upper limit for each epoch. We also derived the upper limit to the brightness temperature in this region to be $9 \times 10^{6} \mathrm{~K}$ at $\lambda 2 \mathrm{~cm}$.

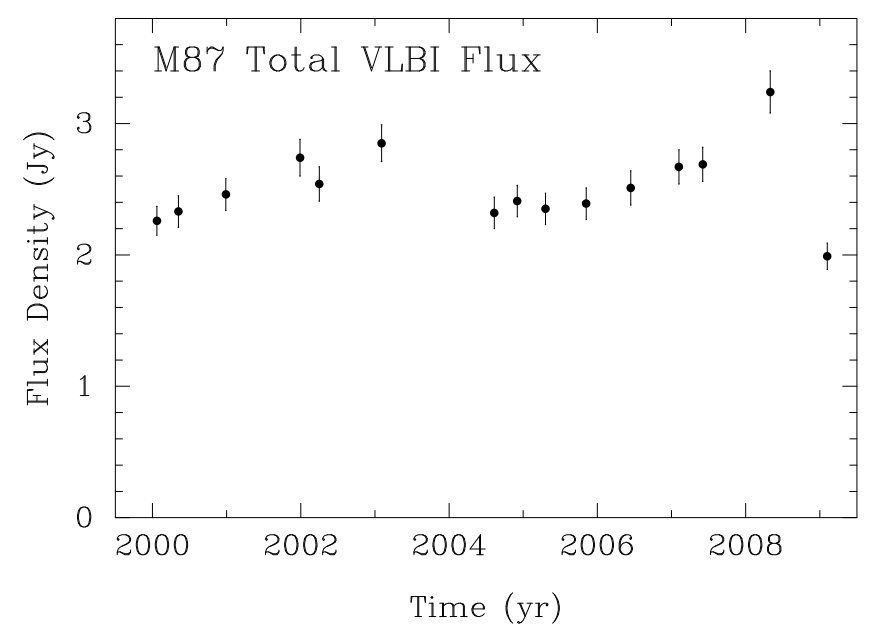

Fig. 3. Total VLBA flux density of M87 at $\lambda 2 \mathrm{~cm}$ versus time.

Table 2. HST-1 $\lambda 20-\lambda 2 \mathrm{~cm}$ spectral index ${ }^{a}$.

\begin{tabular}{ccccc}
\hline \hline \multicolumn{2}{c}{ Epoch } & \multicolumn{2}{c}{$S_{v}[\mathrm{mJy}]$} & $\alpha$ \\
$\lambda 2 \mathrm{~cm}$ & $\lambda 20 \mathrm{~cm}$ & $\lambda 2 \mathrm{~cm}$ & $\lambda 20 \mathrm{~cm}$ & \\
\hline 2005.30 & 2005.35 & $19.7 \pm 1.0$ & $111 \pm 6^{b}$ & $-0.75 \pm 0.03$ \\
2005.85 & 2005.82 & $19.9 \pm 1.0$ & $126 \pm 6^{b}$ & $-0.80 \pm 0.02$ \\
\hline
\end{tabular}

Notes. ${ }^{(a)}$ The spectral index and its errors are formal, see Sect. 4.4; (b) $5 \%$ error assumed for VLBI observations.

\subsection{Spectral properties of HST-1}

Figure 7 superimposes two images of HST-1 at $\lambda 20 \mathrm{~cm}$ (Cheung et al. 2007 and Cheung, priv. comm.) and $\lambda 2 \mathrm{~cm}$ (beam A) for two adjacent epochs. The images were coaligned using the peak of the inner jet as a common reference point. This figure indicates that our observations resolve the HST-1 peak component. We use those epochs to derive the HST-1 spectral properties by producing a rendition of the $\lambda 2 \mathrm{~cm}$ image using the same restoring beam as the $\lambda 20 \mathrm{~cm}$ image, namely, $8 \times 3.4$ mas at a position angle of $0^{\circ}$ (beam B; Fig. 4, right panel). Table 2 lists the epochs of $\lambda 20 \mathrm{~cm}$ and $\lambda 2 \mathrm{~cm}$ observations and the corresponding spectral index $\alpha$, where $S_{v} \propto v^{+\alpha}$. The uncertainties in the spectral indices are formal errors, estimated using standard error propagation methods. When interpreting these values, one has to keep in mind that the resolution of $\lambda 20 \mathrm{~cm}$ and $\lambda 2 \mathrm{~cm}$ are very different. First, the frequencies differ by one magnitude, which produces the small formal error bars of the spectral index. Second, the incomplete $(u, v)$ coverage on short VLBA baselines might explain the missing flux density in the HST-1 region at $\lambda 2 \mathrm{~cm}$ (see Sect. 4.4 for a discussion). The average value of the derived spectral index is $\alpha \sim-0.78$.

\subsection{HST-1 kinematics}

To study the kinematics of HST-1, we fitted the peak of HST-1 and the M 87 core in the image plane with a Gaussian component using IMFIT in AIPS. As shown in Fig. 4, it is difficult to identify moving components in this region because the detections are weak. It is therefore difficult to derive an accurate apparent speed for the HST-1 subcomponents. For this reason, we use the relative position between the peak of HST-1 and the M 87 core to estimate the apparent speed of HST-1. Among the 6 epochs of detection, HST-1 was almost resolved in epoch 2007.10, and appeared to have a double-peak morphology in beam B image 
C. S. Chang et al.: VLBI detection of the HST-1 feature in the M 87 jet at $2 \mathrm{~cm}$

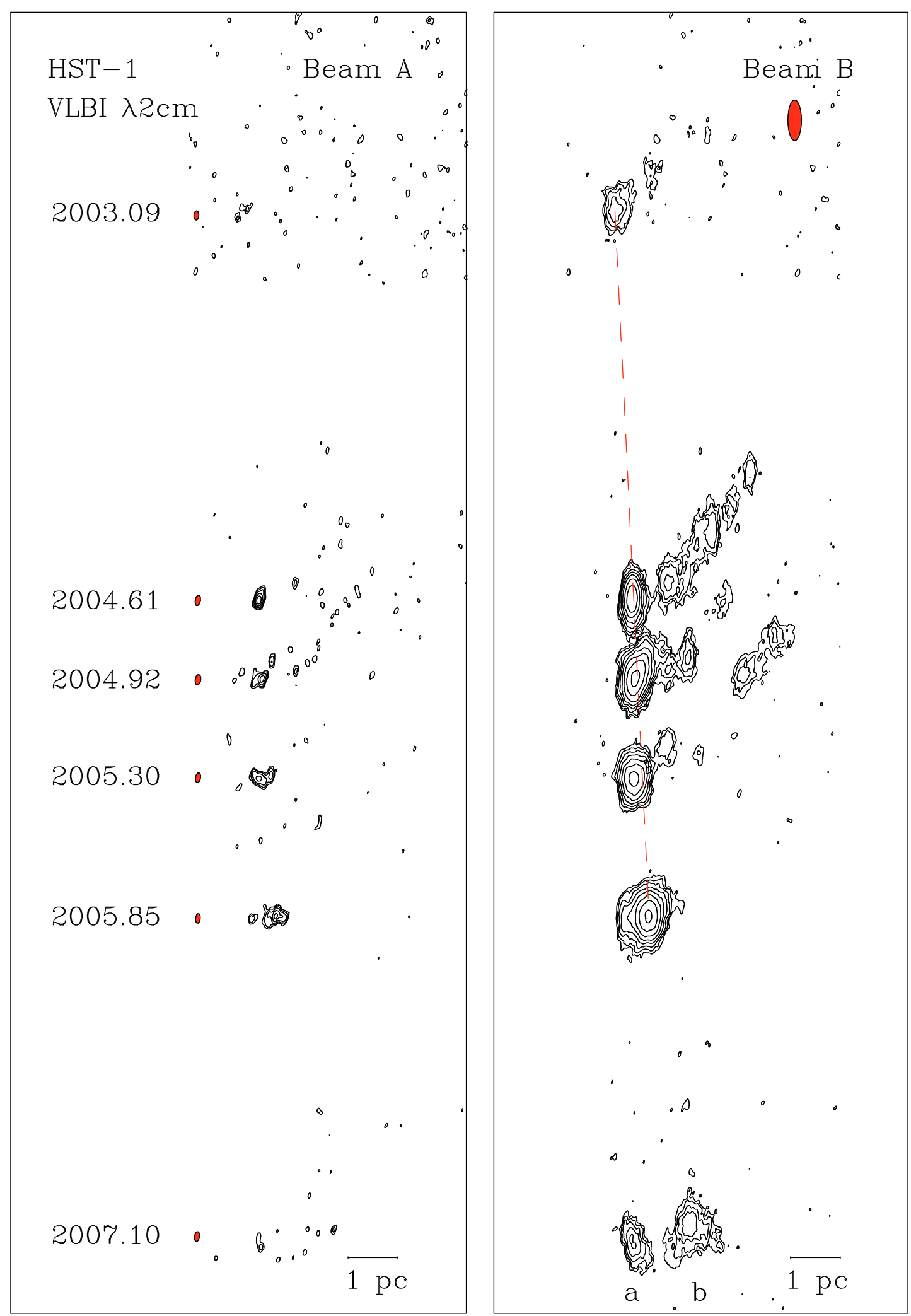

Fig. 4. Images of the HST-1 region restored with beam A (left panel) and beam B (right panel). The distance between epochs is proportional to the relative time interval, and the images are plotted with the same size scale. The contour levels increase by successive factors of $\sqrt{2}$, and the lowest contours for both images are (2003.09 to 2007.10) 0.6, 0.8, 0.8, 0.8, 0.8, and $0.7 \mathrm{mJy}_{\text {beam }}{ }^{-1}$, respectively. The beam sizes of the beam A images are plotted to the right of each epoch label, while the beam size of the beam B images is shown in the upper-right corner. The HST-1 field is phaseshifted -788.5 mas in right ascension (RA) and 348.9 mas in declination (DEC). These images were obtained after performing self-calibration in the imaging process. In the right panel, $\mathbf{a}$ and $\mathbf{b}$ represent the two peaks of HST-1 in epoch 2007.10. The red dashed lines illustrates the linear fit yielding HST-1's projected apparent speed (see Fig. 8). 


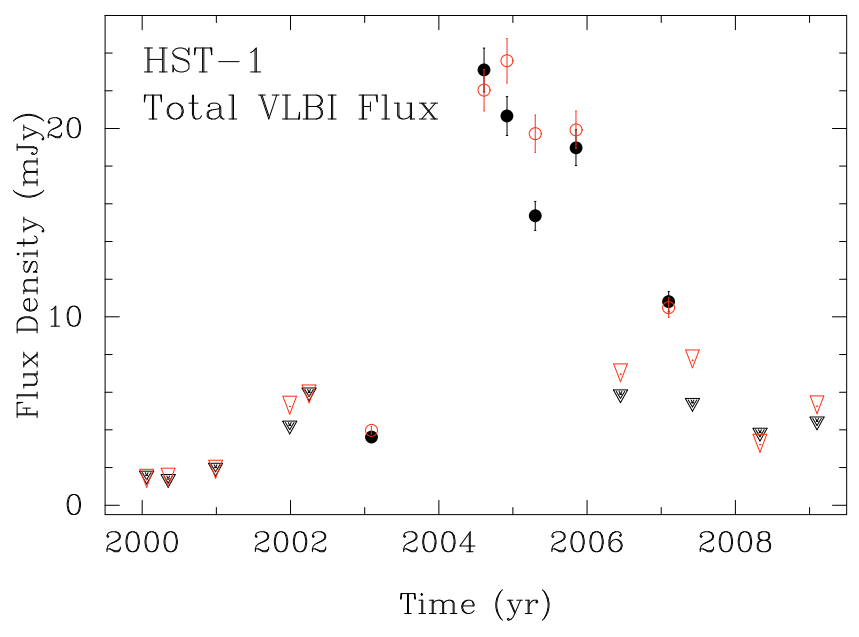

Fig. 5. Total VLBI $\lambda 2 \mathrm{~cm}$ flux density of the HST-1 region versus time. The values for epochs with no confident detection (inverted triangles) are upper limits, whereas the epochs with a detection are marked as dots. The results of using beam A are in black closed circles/triangles, while the results of using beam B are in red open circles/triangles.

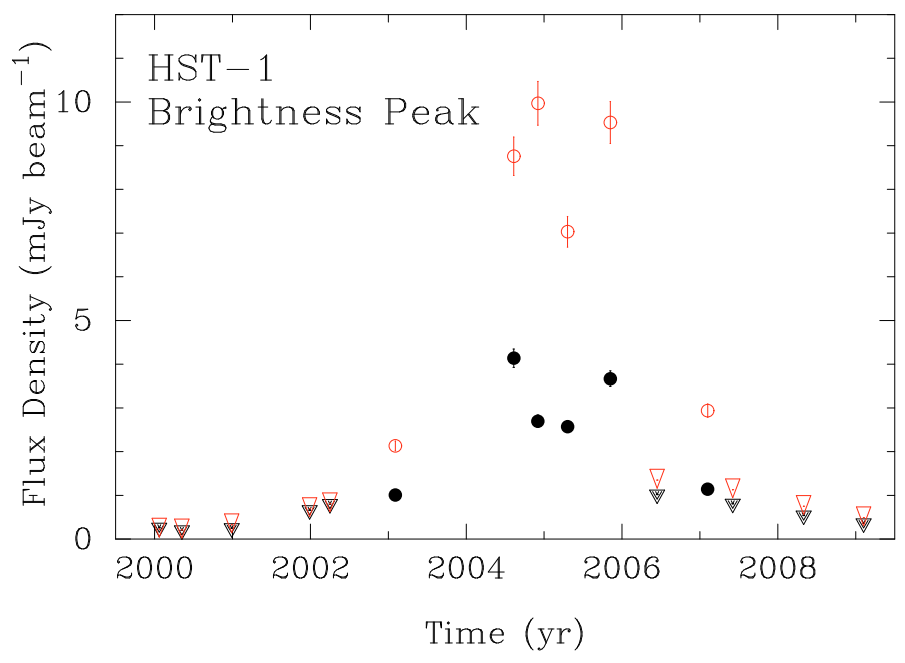

Fig. 6. Brightness peak value at VLBI $\lambda 2 \mathrm{~cm}$ of the HST-1 region versus time. The values for epochs with no confident detection (inverted triangles) are upper limits, whereas the epochs with a detection are marked as dots. The results of using beam A are in black closed circles/triangles, while the results of using beam B are in red open circles/triangles.

(Fig. 4). By considering that the HST-1 detection in 2007.10 was weak, and the double-peak structure might be caused by convolution, we excluded this epoch from the kinematic analysis (see Sect. 4.2 for a discussion). As shown in Fig. 8, we plotted the peak positions of HST- 1 with respect to the M 87 core against time, and we estimated the position errors as the FWHM of the fitted Gaussian component. By applying a linear regression to the peak positions, we obtained a value for the projected apparent speed of the HST-1 feature of $2.36 \pm 1.19 \mathrm{mas} \mathrm{yr}^{-1}$, which corresponds to $\beta_{\text {app }}=0.61 \pm 0.31$.

\section{Discussion}

\subsection{Variability timescale and HST-1 flaring region at parsec-scales}

We derived the variability timescale of the 2005 flare from HST-1 assuming that a single flare would produce a logarithmic
Table 3. HST-1 variability timescale and characteristic size during the rise and fall of the 2005 flare.

\begin{tabular}{lcccc}
\hline \hline Wavelength & $\begin{array}{c}\tau_{\text {rise }} \\
{[\mathrm{yr}]}\end{array}$ & $\begin{array}{c}\tau_{\text {fall }} \\
{[\mathrm{yr}]}\end{array}$ & $\begin{array}{c}\theta_{\text {rise }} \\
{[\mathrm{mas}]}\end{array}$ & $\begin{array}{c}\theta_{\text {fall }} \\
{[\mathrm{mas}]}\end{array}$ \\
\hline VLA $\lambda 2 \mathrm{~cm}$ & 1.5 & 4.9 & $8.7 \delta^{a}$ & $28 \delta$ \\
VLBA $\lambda 2 \mathrm{~cm}$ (beam A) & 1.0 & 4.2 & $5.6 \delta$ & $24 \delta$ \\
VLBA $\lambda 2 \mathrm{~cm}$ (beam B) & 1.0 & 2.7 & $5.7 \delta$ & $15 \delta$ \\
\hline
\end{tabular}

Notes. ${ }^{(a)}$ The characteristic size is related to the Doppler factor $\delta$.

rise and fall in the light curve. By fitting the available light curves from VLBI and VLA measurements before and after the maximum flux density during the flare, we derived the corresponding variability timescales and the upper limit to the characteristic size of the region in which the flare was produced. We define the logarithmic variability timescale as $\tau_{\text {var }}=\mathrm{d} t / \mathrm{d}[\ln (S)]$, where $S$ is the flux density and $t$ is the time interval between observations in units of years (Burbidge et al. 1974). We estimate the upper limit to the characteristic size of the emission region from lighttravel time $\theta_{\text {char }} \approx 0.13(1+z) D^{-1} \tau_{\text {var }} \delta$, where $z$ is the redshift, $D$ is the luminosity distance in Gpc, and $\delta$ is the Doppler factor (Marscher et al. 1979). Table 3 shows the result of the derived characteristic size of the 2005 flare in HST-1.

Harris et al. (2003) estimated the Doppler factor $\delta$ of HST-1 to have a value between 2 and 5, based on decay timescales of Chandra observations. In the same context, Wang \& Zhou (2009) estimated the Doppler factor of HST-1 to be $3.57 \pm 0.51$ by fitting the non-simultaneous spectral energy distribution of M87 using a synchrotron spectrum model. Simulations incorporating MHD models for the M 87 jet have suggested that the recollimation shock formed close to the HST-1 position had a relatively low Doppler factor $\sim 1-2$ (Gracia et al. 2009). If we were to assume that $\delta=3.57$, the derived characteristic sizes of HST-1 emission region during its flaring time would be $20<\theta_{\text {rise }}<31$ and $54<\theta_{\text {fall }}<100$ mas. The size scale of structural changes of HST-1 (see Fig. 4) is in the range 20-50 mas ( 1 pc $=12.5$ mas), which is within the derived characteristic size scale. However, if $\delta$ were lower than 3.5, $\theta_{\text {rise }}$ derived from VLBA $\lambda 2 \mathrm{~cm}$ would be smaller than 20 mas, creating a causality problem, since the largest structural change of HST-1 cannot be greater than the upper limit to the information propagation time. Therefore, $\delta>3.5$ is required because of the causality argument.

If $\delta$ were lower than 3.5 in the HST-1 region, we could explain the causality problem using the self-calibration procedure that we used while imaging HST-1. We applied self-calibration to the inner jet and HST-1 for all of the epochs with detections in beam A and beam B. Self-calibration is more effective for stronger objects. In our case, the marginal detection in epoch 2003.09 might not permit us to recover extended emission, in contrast to stronger detections between 2004.61 and 2005.85, in which after self-calibration, we were able to recover more extended emission.

\subsection{Speeds of HST-1}

We used the peak position and fitted Gaussian errors to estimate the projected apparent speed of HST- $1 \beta_{\text {app }}=0.61 \pm 0.31$ (see Fig. 8), which is sub-luminal. In the kinematic analysis, we excluded epoch 2007.10 to ensure a robust kinematic result. However, to make our results more solid, we estimated the upper and lower limit to the HST-1 apparent speed by including epoch 2007.10 as a test. The derived possible range of the apparent 

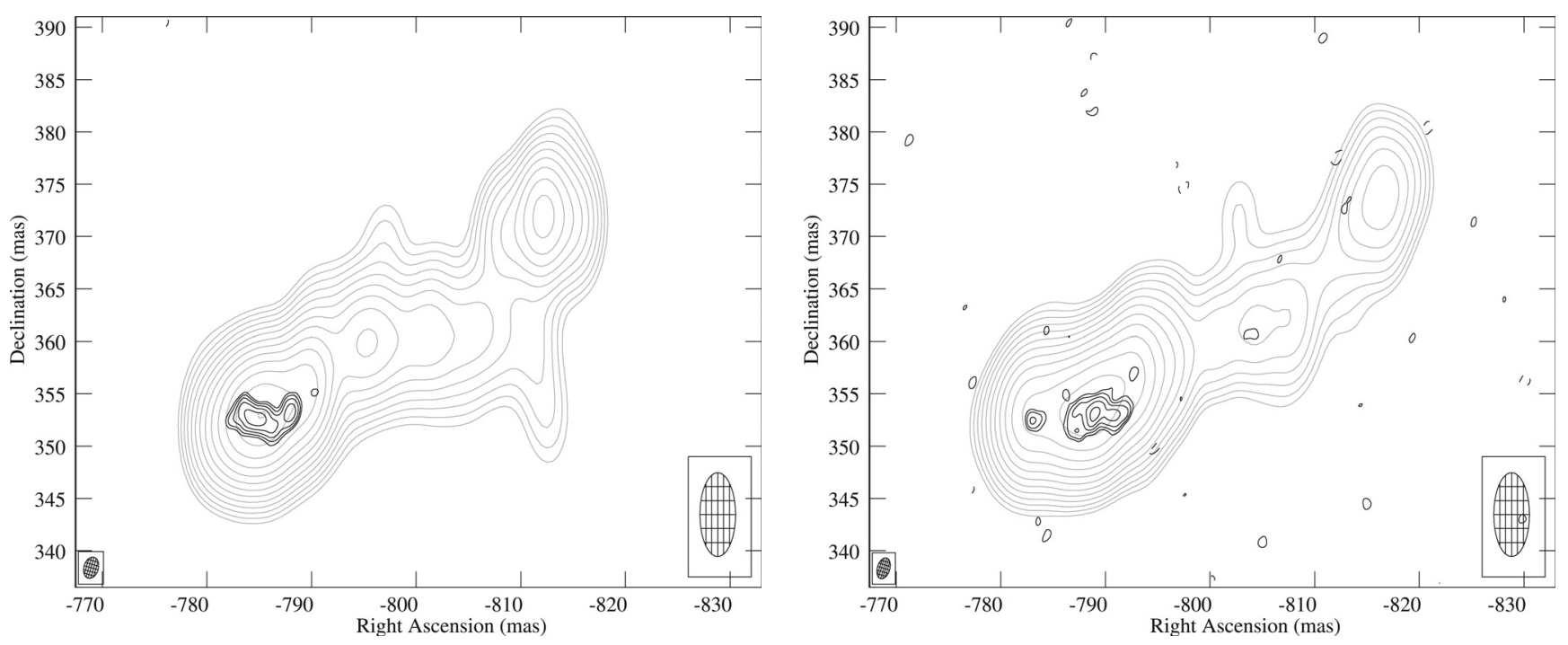

Fig. 7. VLBA images of the HST-1 region in M 87 at $\lambda 2 \mathrm{~cm}$ (this paper: black contour, beam size at half power level $=2 \times 1 \mathrm{mas}, \mathrm{PA}=-16^{\circ}$, plotted bottom left) and $\lambda 20 \mathrm{~cm}$ (Cheung et al. (2007): gray contour, beam size $=8 \times 3.4$ mas, $\mathrm{PA}=0^{\circ}$, plotted bottom right). Left-hand panel: epoch $2005.30\left(\lambda 2 \mathrm{~cm}\right.$, peak surface brightness: 3.4 mJy beam $\left.^{-1}\right)$ and $2005.35\left(\lambda 20 \mathrm{~cm}\right.$, peak: $45 \mathrm{mJy}$ beam $\left.{ }^{-1}\right)$; right-hand panel: epoch 2005.85 $\left(\lambda 2 \mathrm{~cm}\right.$, peak: $\left.3.3 \mathrm{mJy}_{\text {beam }}^{-1}\right)$ and $2005.82\left(\lambda 20 \mathrm{~cm}\right.$, peak: $42 \mathrm{mJy}$ beam $\left.^{-1}\right)$. The lowest contour is $0.7 \mathrm{mJy}$ beam ${ }^{-1}$, and the contour levels are separated by a factor of $\sqrt{2}$. The images of two frequencies were registered using the peak of the inner jet of HST-1 as a common reference point.

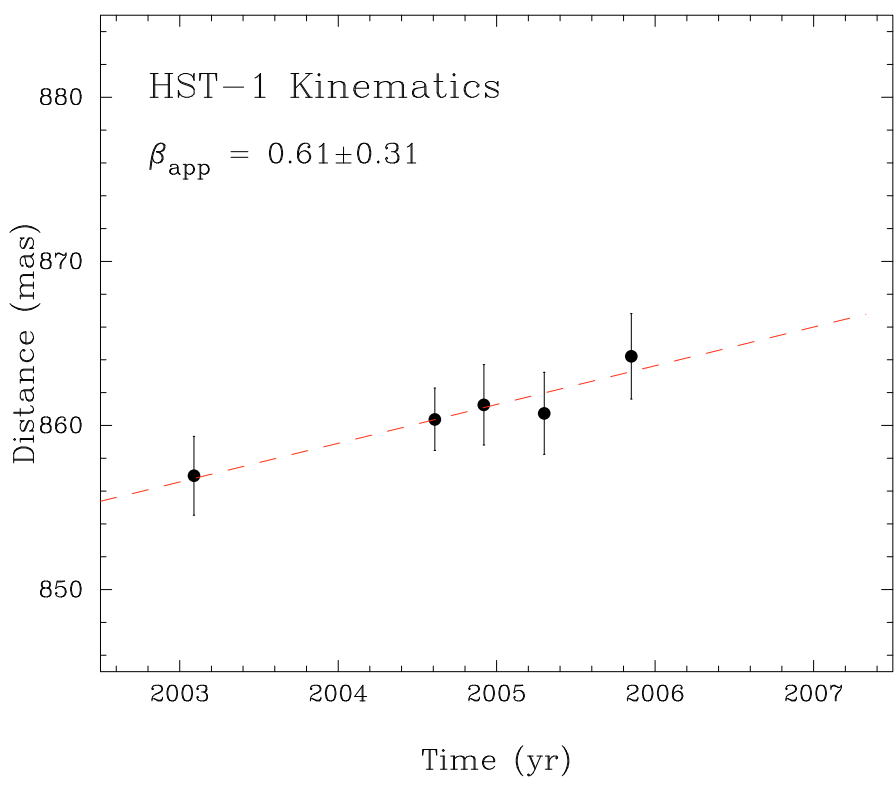

Fig. 8. The linear fit of HST-1 proper motion shown as the red dashed line. This plot illustrates the position of HST-1 component peaks with respect to the M 87 core component as a function of time from 2003.09 to 2005.85 . The fitted projected apparent speed of HST- $1 \beta_{\text {app }}$ is shown at the upper-left corner of the plot.

speed is $0.23<\beta_{\text {app }}<1.2$, which is still consistent with mildly relativistic jet motion.

To compare our kinematic results with previous findings, we show the positional evolution on the sky of the HST-1 peak at VLBA $\lambda 2 \mathrm{~cm}$ with both beam A and beam B (see Fig. 4), and components $\mathrm{C} 1$ and C2 at VLBA $\lambda 20 \mathrm{~cm}$ (Cheung et al. 2007, and priv. comm.). As illustrated, our derived apparent speed range and structural evolution in time at $\lambda 2 \mathrm{~cm}$ are consistent with the $\lambda 20 \mathrm{~cm}$ results. However, our subluminal speed measurements of HST-1 are inconsistent with the high apparent superluminal motions reported in Cheung et al. (2007) from
VLBA $\lambda 20 \mathrm{~cm}$ observations and with the HST (Biretta et al. 1999). Nevertheless, in the VLBA $\lambda 20 \mathrm{~cm}$ observations (Cheung et al. 2007), lower speeds were derived from some components in HST-1: $\mathrm{c} 2$ has $\beta_{\text {app }}=0.47 \pm 0.39$, and HST-1d has $\beta_{\text {app }}=1.14 \pm 0.14$. Our results are consistent with these findings.

\subsection{Detection limits}

HST-1 was not detected in epoch 2006.45. This epoch had only 22 minute integration time and the sampling rate was $128 \mathrm{Mbit} \mathrm{s}^{-1}$. Therefore, the rms level $\left(0.35 \mathrm{mJy} \mathrm{beam}^{-1}\right)$ was not low enough to detect HST-1, which was also fading according to our light curve (Fig. 5).

The total flux density of HST-1 measured by the VLA at $\lambda 2 \mathrm{~cm}$ reached its maximum value of $\sim 123 \mathrm{mJy}$ in 2005 (Harris et al. 2006). Our VLBA $\lambda 2 \mathrm{~cm}$ results recovered a HST- 1 total flux density of $\sim 23 \mathrm{mJy}$, which is only $19 \%$ of the VLA measurement. We conclude that the innermost region of HST-1 was resolved by using the long baselines of the VLBA. The low measured flux density suggests that HST-1 is very extended.

\subsection{HST-1 parsec-scale spectrum}

We derived the spectral index of the HST-1 region based on VLBA $\lambda 2 \mathrm{~cm}$ and $\lambda 20 \mathrm{~cm}$ data (Table 2). However, one should be cautious with these values. First, the two datasets have a frequency difference of a factor of 10, which results in small formal error bars in the derived spectral index. The spectral index here could infer a trend in the spectrum of HST-1 and the physical condition of the region, but not the absolute value. Second, we found that the incomplete $(u, v)$ coverage on short VLBA baselines has resulted in missing flux in the HST-1 region at $\lambda 2 \mathrm{~cm}$. Therefore, the spectral index value of HST-1 region is a lower limit, and the results suggest that HST-1 was optically thin during the flare in 2005. If we were to assume that HST-1 is a flatspectrum source, there would have been $\sim 90 \mathrm{mJy}$ missing flux in this region in our $2 \mathrm{~cm}$ VLBA measurements. 


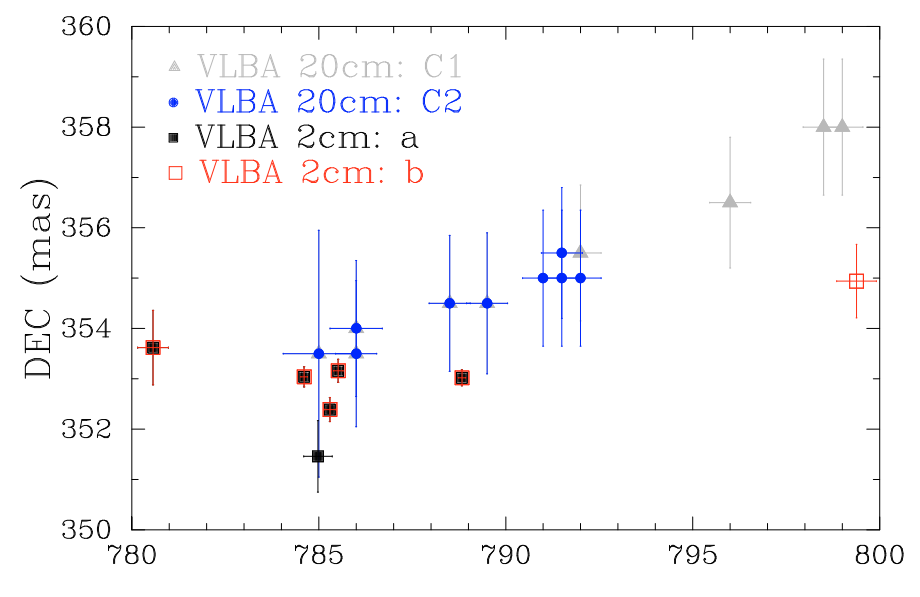

RA (mas)

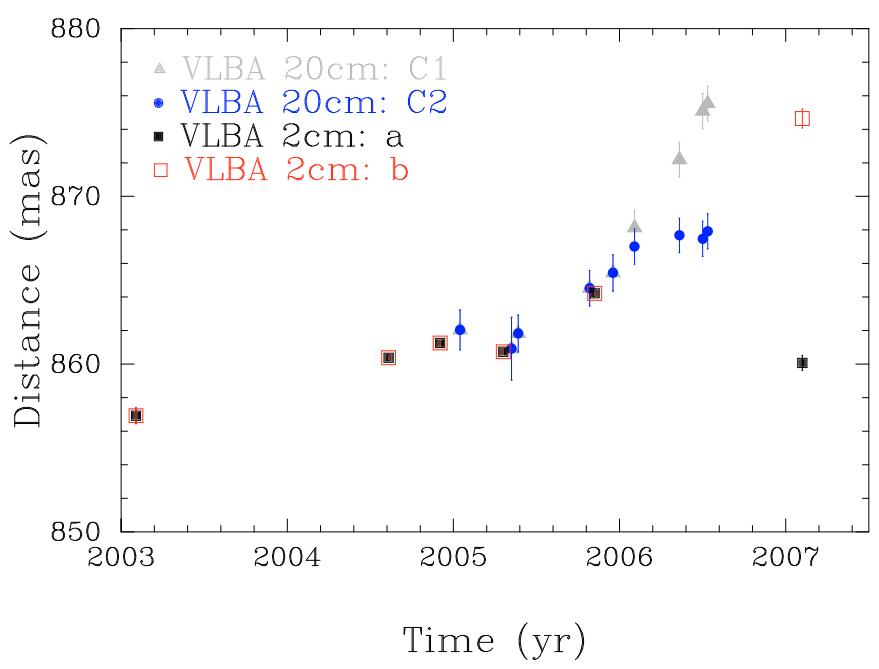

Time $(\mathrm{yr})$

Fig. 9. HST-1 sky positions (left) and radial distance as a function of time (right) relative to the M 87 core. VLBA $\lambda 20 \mathrm{~cm}$ (Cheung et al. 2007 , priv. comm.): time range of components $\mathrm{C} 1$ and $\mathrm{C} 2$ is from 2005.04 to 2006.53 ; VLBA $\lambda 2 \mathrm{~cm}$ (beam B): time range of components $\mathbf{a}$ and $\mathbf{b}$ is from 2003.09 to 2007.10; components $\mathbf{a}$ and $\mathbf{b}$ are shown in Fig. 4.

\subsection{A blazar nature of HST-1}

The intensity of HST-1 reached a maximum in different wavebands in 2005, and the light curves from the VLA $\lambda 2 \mathrm{~cm}$, VLBA $\lambda 20 \mathrm{~cm}, \mathrm{NUV}$, and X-ray observations all show the same tendency (Harris et al. 2009; Madrid 2009). Our light curve of HST-1 at VLBA $\lambda 2 \mathrm{~cm}$ (Fig. 5) is consistent with the other observations. Although this trend can be interpreted as evidence that HST-1 is the source of the HESS-detected TeV flare in 2005 (Aharonian et al. 2006), its VLBA radio properties that we derived do not support this idea, e.g. (i) the very low compactness of its dominant emission; (ii) its low brightness temperature; (iii) its sub-luminal motion; and (iv) the possibly low optical depth across the feature at parsec-scales. Those are in contrast to the typical blazar core features, which tend to have flat or inverted spectral indices in cm-wave VLBA images. We observed that HST-1 has radio properties consistent with those commonly seen in jet components. TeV observatories are unable to resolve the M 87 core and HST-1 separately. To probe the origin of $\mathrm{TeV}$ emission, searching for correlations between variability for different bands is a powerful tool. This approach was performed successfully for a sample of blazars by Kovalev et al. (2009), and for M 87 by Acciari et al. (2009). Another means of probing the $\mathrm{TeV}$ origin is to apply physical models to multiwavelength observations. Applying models has helped to uncover radio-TeV connections. For example, high energy flares could be generated from parsec-scale radio jets by inverse Compton scattering of the photons and particles being emitted from the core, and Stawarz et al. (2006) used this approach to explain the TeV flare from M87 in 2005. However, Acciari et al. (2009) observed the VHE flare of M87 in 2008, and found that there might be correlations with the radio flare detected by VLBA at $43 \mathrm{GHz}$ from the core. From our results, although we cannot fully discount, however, that HST-1 was the source of the TeV flare in 2005 based on our results alone, we do not agree that HST-1 has a blazar nature, as suggested by Harris et al. (2008).

\section{Summary}

By analyzing our VLBA $\lambda 2 \mathrm{~cm}$ data from 2000.06 to 2009.10, we have detected HST-1 during 2003.09-2007.10, which covered the multi-band flaring period of HST-1. The total flux density of HST-1 varied within the range 4-24 mJy for our detections. By comparing the images of VLBA $\lambda 2 \mathrm{~cm}$ and $\lambda 20 \mathrm{~cm}$, we measured a steep spectrum with $\alpha>-0.8$ in this region. The projected apparent speed of HST-1 derived from the brightness peak position is $0.61 \pm 0.31 c$, which implies a subluminal motion at VLBA $\lambda 2 \mathrm{~cm}$.

Our results have indicated that HST-1 is extremely extended on parsec scales, and has a steep spectrum. No compact feature with a brightness temperature higher than $9 \times 10^{6} \mathrm{~K}$ is present in the $\lambda 2 \mathrm{~cm}$ VLBA observations of this region of the M 87 jet, which implies that HST-1 does not have the properties of a standard blazar core. Combining our findings, we do not find evidence that HST-1 in the jet of M 87 has a blazar nature.

Acknowledgements. We thank A. Moré, G. Cimò, S. Mühle, M. A. Garrett, R. W. Porcas, R. C. Walker, and C. M. Fromm for valuable comments and inspiring discussions. Special thanks are due to C. C. Cheung for providing the VLBA $\lambda 20 \mathrm{~cm}$ images (Cheung et al. 2007), and D. E. Harris for providing VLA $\lambda 2 \mathrm{~cm}$ light curve (Harris et al. 2009). We thank the anonymous referee for useful comments and suggestions. This research was supported by the EU Framework 6 Marie Curie Early Stage Training program under contract number MEST/CT/2005/19669 "ESTRELA". C.S. Chang is a member of the International Max Planck Research School for Astronomy and Astrophysics. Part of this project was done while Y.Y.K. was working as a research fellow of the Alexander von Humboldt Foundation. Y.Y.K. was partly supported by the Russian Foundation for Basic Research (project 08-02-00545) and the Alexander von Humboldt return fellowship. This research has made use of data from the $2 \mathrm{~cm}$ Survey (Kellermann et al. 2004) and MOJAVE programs that is maintained by the MOJAVE team (Lister et al. 2009a). The MOJAVE project is supported under National Science Foundation grant AST-0807860 and NASA Fermi grant NNX08AV67G. Part of this work made use of archived VLBA and VLA data obtained by K. I. Kellermann, J. Biretta, F. Owen, and W. Junor. The Very Long Baseline Array is operated by the USA National Radio Astronomy Observatory, which is a facility of the USA National Science Foundation operated under cooperative agreement by Associated Universities, Inc. This research has made use of the NASA/IPAC Extragalactic Database (NED) which is operated by the Jet Propulsion Laboratory, California Institute of Technology, under contract with the National Aeronautics and Space Administration. This research has made use of NASA's Astrophysics Data System.

\section{References}

Abdo, A. A., Ackermann, M., Ajello, M., et al. 2009, ApJ, 707, 55 Acciari, V. A., Aliu, E., Arlen, T., et al. 2009, Science, 325, 444

Aharonian, F., Akhperjanian, A. G., Bazer-Bachi, A. R., et al. 2006, Science, 314,1424

Baade, W. 1956, ApJ, 123, 550

Biretta, J. A., Sparks, W. B., \& Macchetto, F. 1999, ApJ, 520, 621

Burbidge, G. R., Jones, T. W., \& Odell, S. L. 1974, ApJ, 193, 43 
C. S. Chang et al.: VLBI detection of the HST-1 feature in the M 87 jet at $2 \mathrm{~cm}$

Cheung, C. C., Harris, D. E., \& Stawarz, Ł. 2007, ApJ, 663, L65

Curtis, H. D. 1918, Publications of Lick Observatory, 13, 31

Gracia, J., Vlahakis, N., Agudo, I., Tsinganos, K., \& Bogovalov, S. V. 2009, ApJ, 695, 503

Harms, R. J., Ford, H. C., Tsvetanov, Z. I., et al. 1994, ApJ, 435, L35

Harris, D. E., Biretta, J. A., Junor, W., et al. 2003, ApJ, 586, L41

Harris, D. E., Cheung, C. C., Biretta, J. A., et al. 2006, ApJ, 640, 211

Harris, D. E., Cheung, C. C., Stawarz, L., et al. 2008, in Extragalactic Jets: Theory and Observation from Radio to Gamma Ray, ed. T. A. Rector \& D. S. De Young, ASP Conf. Ser., 386, 80

Harris, D. E., Cheung, C. C., Stawarz, Ł., Biretta, J. A., \& Perlman, E. S. 2009, ApJ, 699, 305

Jordán, A., Côté, P., Blakeslee, J. P., et al. 2005, ApJ, 634, 1002

Kellermann, K. I., Lister, M. L., Homan, D. C., et al. 2004, ApJ, 609, 539

Komatsu, E., Dunkley, J., Nolta, M. R., et al. 2009, ApJS, 180, 330

Kovalev, Y. Y., Kellermann, K. I., Lister, M. L., et al. 2005, AJ, 130, 2473

Kovalev, Y. Y., Lister, M. L., Homan, D. C., \& Kellermann, K. I. 2007, ApJ, 668, L27

Kovalev, Y. Y., Aller, H. D., Aller, M. F., et al. 2009, ApJ, 696, L17
Lister, M. L., Aller, H. D., Aller, M. F., et al. 2009a, AJ, 137, 3718

Lister, M. L., Cohen, M. H., Homan, D. C., et al. 2009b, AJ, 138, 1874

Lobanov, A. P. 1998, A\&A, 330, 79

Ly, C., Walker, R. C., \& Junor, W. 2007, ApJ, 660, 200

Madrid, J. P. 2009, AJ, 137, 3864

Marscher, A. P. 2008, in Extragalactic Jets: Theory and Observation from Radio to Gamma Ray, ed. T. A. Rector \& D. S. De Young, ASP Conf. Ser., 386, 437 Marscher, A. P., Marshall, F. E., Mushotzky, R. F., et al. 1979, ApJ, 233, 498

Petrov, L., Kovalev, Y. Y., Fomalont, E. B., \& Gordon, D. 2008, AJ, 136, 580

Petrov, L., Gordon, D., Gipson, J., et al. 2009, J. Geod., 83, 859

Seyfert, C. K. 1943, ApJ, 97, 28

Shklovsky, I. S. 1964, Soviet Astron., 7, 748

Stawarz, Ł., Aharonian, F., Kataoka, J., et al. 2006, MNRAS, 370, 981

Ulvestad, J. S., \& Wrobel, J. M. 2009, VLBA status summary, http://www. vlba.nrao.edu/astro/obstatus/current/obssum.html, NRAO Urry, C. M., \& Padovani, P. 1995, PASP, 107, 803

Walker, R. C., Ly, C., Junor, W., \& Hardee, P. J. 2008, J. Phys. Conf. Ser., 131, 012053

Wang, C., \& Zhou, H. 2009, MNRAS, 395, 301 